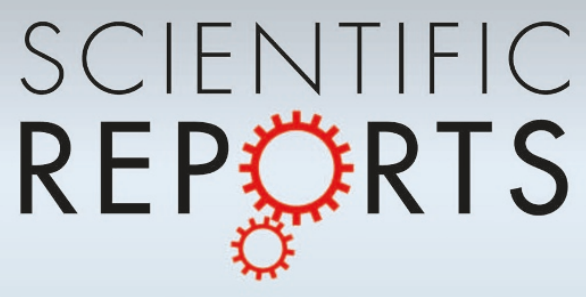

OPEN

SUBJECT AREAS:

TWO-DIMENSIONAL

MATERIALS

ELECTRONIC DEVICES

MECHANICAL PROPERTIES

ELECTRONIC PROPERTIES AND

MATERIALS

Received

15 October 2012

Accepted

29 May 2013

Published

18 June 2013

Correspondence and requests for materials should be addressed to

S.-W.K. (kimsw1@ skku.edu)

\title{
Piezoelectric two-dimensional
}

\section{nanosheets/anionic layer heterojunction for efficient direct current power generation}

\author{
Kwon-Ho Kim' ', Brijesh Kumar', Keun Young Lee', Hyun-Kyu Park', Ju-Hyuck Lee², Hyun Hwi Lee ${ }^{3}$, \\ Hoin Junn ${ }^{4}$, Dongyun Lee ${ }^{4} \&$ Sang-Woo Kim ${ }^{1,2}$
}

\begin{abstract}
'School of Advanced Materials Science and Engineering, Sungkyunkwan University (SKKU), Suwon 440-746, Republic of Korea, ${ }^{2}$ SKKU Advanced Institute of Nanotechnology (SAINT), Center for Human Interface Nanotechnology (HINT), Sungkyunkwan University (SKKU), Suwon 440-746, Republic of Korea, ${ }^{3}$ Pohang Accelerator Laboratory, POSTECH, Pohang 790-784, Republic of Korea, ${ }^{4}$ Department of Advanced Integrated Circuit, Department of Nanofusion Engineering, College of Nanoscience and Nanotechnology, Pusan National University, Busan, 609-735, Republic of Korea.
\end{abstract}

Direct current (DC) piezoelectric power generator is promising for the miniaturization of a power package and self-powering of nanorobots and body-implanted devices. Hence, we report the first use of two-dimensional (2D) zinc oxide ( $\mathrm{ZnO}$ ) nanostructure and an anionic nanoclay layer to generate piezoelectric DC output power. The device, made from 2D nanosheets and an anionic nanoclay layer heterojunction, has potential to be the smallest size power package, and could be used to charge wireless nano/micro scale systems without the use of rectifier circuits to convert alternating current into DC to store the generated power. The combined effect of buckling behaviour of the $\mathrm{ZnO}$ nanosheets, a self-formed anionic nanoclay layer, and coupled semiconducting and piezoelectric properties of $\mathrm{ZnO}$ nanosheets contributes to efficient $\mathrm{DC}$ power generation. The networked $\mathrm{ZnO}$ nanosheets proved to be structurally stable under huge external mechanical loads.

\footnotetext{
C onsidering the low power consumption features of various portable devices, sensors, and wireless bodyimplantable devices as well as the need to reduce global dependency on energy sources based on fossil fuels, the concept of power generation through energy harvesting from environmental sources has gained new relevance. Recent advances in piezoelectric power generators open numerous doors for meaningful power generation through environmentally abundant mechanical energy harvesting for practical applications, particularly, for self-powered and low power-consuming devices ${ }^{1-7}$. Most piezoelectric power generators are based on alternating current $(\mathrm{AC})$ signal generation ${ }^{1-3,8-11}$, so rectifier circuits should be needed to convert AC power into direct current (DC) power for storing the generated power, resulting in increased total sizes of the power packages. Furthermore, in the process of converting, power is drastically reduced.

In spite of these problems, no researches on the realization of piezoelectric DC power generators using typical piezoelectric energy harvesting materials such as lead zirconate titanate, polyvinylidene fluoride, and barium titanate have been reported ${ }^{10,12-16}$. Recently, although DC output piezoelectric nanogenerators based on nanostructures of zinc oxide $(\mathrm{ZnO})$ with piezoelectric and semiconducting coupled properties have been demonstrated via lateral mechanical compression of bent nanowires or nanorod $s^{17}$, the power-generating performance is still too low for practical applications. In addition, previous DC output nanogenerators have been based on onedimensional (1D) $\mathrm{ZnO}$ nanowires and nanorods ${ }^{17-19}$. Superior piezoelectric properties are expected only in the case of $1 \mathrm{D}$ nanostructures with an extremely high aspect ratio since the piezoelectric coefficient dramatically increases with increment of the aspect ratio of $1 \mathrm{D}$ nanostructure, whereas mechanical instability should be resolved because the DC output is obtained only via lateral mechanical bending.

In the last few years, emerging layered 2D nanomaterials such as graphene, boron nitride nanosheets, and molybdenum disulfide nanosheets have attracted much attention due to their novel and unique physical and chemical properties that are different from the bulk form ${ }^{20-22}$. Semiconducting $2 \mathrm{D}$ nanostructures such as nanosheets, nanoplates, and nanowalls also have many attractive properties such as nanometer scale thickness,
} 
high surface-to-volume ratio, and good mechanical durability for applications in energy conversion and storage devices, chemical and biological sensors, etc $\mathrm{c}^{23,24}$. In spite of these advantages, studies regarding $2 \mathrm{D}$ nanostructure-based devices are less common compared to those regarding $1 \mathrm{D} \mathrm{ZnO}$ nanostructure-based devices. Especially, there has been no report on 2D nanostructure-based piezoelectric nanogenerators.

Previously, extensive studies have been conducted on the synthesis of layered double hydroxide ( $\mathrm{LDH})$ films or coating on different substrate for developing novel applications including monolithic catalysis, anti-corrosion coatings, modified electrodes, components in optical or magnetic devices, and sensors ${ }^{25-30}$. LDH acts as an anionic clay, anion scavenger and exchanger, and a capacitor with weak conductivity. In this work, it was found that $\mathrm{LDH}$ as an anionic clay plays an important role in generating DC output from piezoelectric nanogenerators. $2 \mathrm{D} \mathrm{ZnO}$ nanosheets/LDH networks are synthesized on aluminum (Al) electrode. Subsequently, 2D $\mathrm{ZnO}$ nanosheet-based piezoelectric DC nanogenerators with highly efficient output performance are fabricated, which have great potential for use in portable electronics and further miniaturization of a power packages. Furthermore, compared to previous piezoelectric DC output nanogenerators, the $\mathrm{ZnO}$ nanosheet-based piezoelectric nanogenerator has high and stable power generation performance because of its structural stabilization. From nanoindentation measurements, the mechanical durability of the $2 \mathrm{D} \mathrm{ZnO}$ nanosheets is found to be excellent compared to $1 \mathrm{D} \mathrm{ZnO}$ nanorods.

\section{Results}

Formation of piezoelectric 2D $\mathrm{ZnO}$ nanosheets/anionic layer heterojunction. Figure $1 \mathrm{a}$ and $1 \mathrm{~b}$ show field emission scanning electron microscopy (FE-SEM) and cross-sectional transmission electron microscopy (TEM) images of $\mathrm{ZnO}$ nanosheet networks grown on the Al electrode, respectively. The $\mathrm{ZnO}$ nanosheet networks are slightly curved and have an uneven surface morphology on a large scale. The observed average width and height of the $\mathrm{ZnO}$ nanosheets are about $80 \mathrm{~nm}$ and $3 \mu \mathrm{m}$, respectively (see Supplementary Fig. S1). A lattice spacing of $0.52 \mathrm{~nm}$ between adjacent lattice planes of a dispersed $\mathrm{ZnO}$ nanosheet is calculated from high-resolution (HR) TEM image, which confirms that the preferred growth direction of the $\mathrm{ZnO}$ nanosheets is [001] (Fig. 1c). Anisotropic growth of the $\mathrm{ZnO}$ nanosheet along the [001] direction is due to the inherent polar properties of $\mathrm{ZnO}$ along the c-axis. Further, a fast Fourier transformation (FFT) of the dispersed $\mathrm{ZnO}$ nanosheet in the inset shows that $\mathrm{ZnO}$ nanosheets grown along the [001] direction are single crystalline.

In order to investigate the interface between the $\mathrm{ZnO}$ nanosheets and the $\mathrm{Al}$ electrode, micro energy-dispersive spectroscopy ( $\mu$-EDS) measurements were carried out. The $\mu$-EDS profile from the point marked " $x$ " (in a cross-sectional TEM image of an inset) is shown in Supplementary Fig. S2a. In addition to strong peaks from oxygen and $\mathrm{Al}$, weak peaks corresponding to $\mathrm{Zn}$ are also observed, indicating the formation of $\mathrm{Zn}$ and $\mathrm{Al}$ mixed oxide and $\mathrm{ZnAl}: \mathrm{LDH}$ at the interface between the $\mathrm{ZnO}$ nanosheets and the $\mathrm{Al}$ electrode. Further, the synchrotron X-ray diffraction (XRD) pattern of the $\mathrm{ZnO}$ nanosheets network grown on the $\mathrm{Al}$ electrode confirms the formation of $\mathrm{ZnAl}: \mathrm{LDH}$ at the interface of $\mathrm{ZnO}$ nanosheets and $\mathrm{Al}$ (Fig. 1d). In addition to diffraction peaks from the hexagonal $\mathrm{ZnO}$ (002), (100), and (101) planes, we observed three additional strong peaks at $22.7^{\circ}$, $33.6^{\circ}$, and $60.1^{\circ}$ corresponding to $\mathrm{ZnAl}: \mathrm{LDH}$ planes, which is confirmed from standard powder diffraction peaks of $\mathrm{ZnAl}: \mathrm{LDH}$ (JCPDS card No. 85-1327). Furthermore, micro-Raman spectroscopy confirms the formation of $\mathrm{ZnAl}: \mathrm{LDH}$ at the interface of a

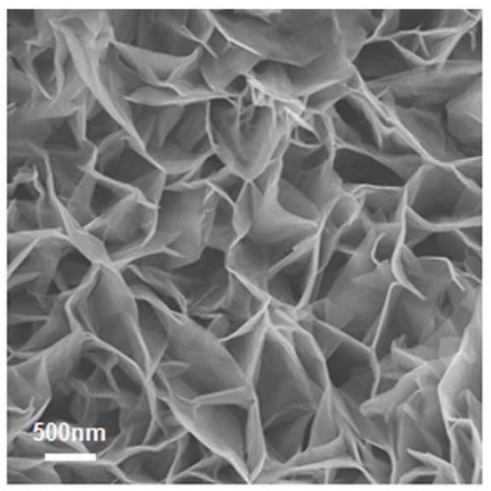

C

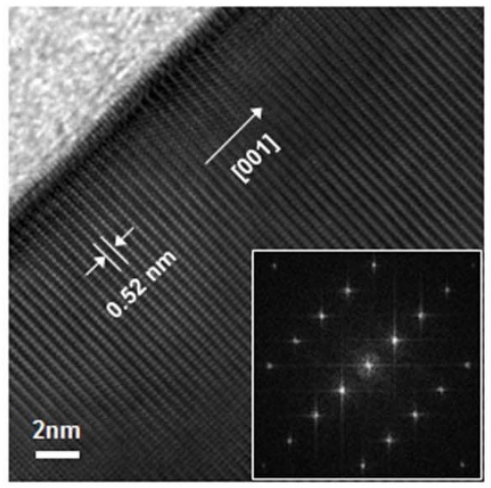

b
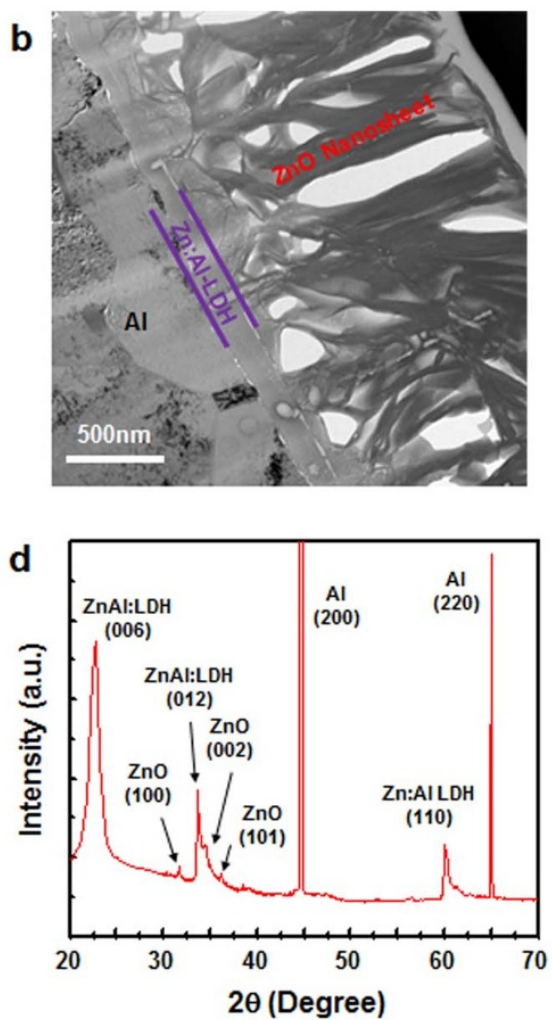

Figure $1 \mid$ Characterization of the ZnO nanosheets. a, Plan-view FE-SEM image of the ZnO nanosheets network grown on Al. b, Cross-sectional TEM image of the $\mathrm{ZnO}$ nanosheets network, formation of $\mathrm{LDH}$ at the interface between the $\mathrm{ZnO}$ nanosheets and the $\mathrm{Al}$ electrode is highlighted. c, $\mathrm{HR}$ TEM image of a dispersed $\mathrm{ZnO}$ nanosheet and the inset is a corresponding FFT pattern. A lattice spacing of $0.52 \mathrm{~nm}$ between adjacent lattice planes of a dispersed $\mathrm{ZnO}$ nanosheet confirms the preferred growth direction of the $\mathrm{ZnO}$ nanosheets is [001]. FFT of the dispersed $\mathrm{ZnO}$ nanosheet in the inset shows single crystalline nature. $\mathrm{d}$, Synchrotron XRD pattern of the $\mathrm{ZnO}$ nanosheets network as grown on the Al foil. 
$\mathrm{ZnO}$ nanosheets and $\mathrm{Al}$ (see Supplementary Fig. S2b). A peak at $1384 \mathrm{~cm}^{-1}$ is attributed to interlayer nitrate anions $\left(\mathrm{NO}_{3}{ }^{-}\right)$in the LDH layer ${ }^{29}$.

Electrical output of a piezoelectric nanogenerator fabricated with 2D $\mathrm{ZnO}$ nanosheets/anionic layer heterojunction. In order to fabricate piezoelectric nanogenerators, gold $(\mathrm{Au})$-coated polyethersulphone (PES) as a top electrode is placed above the $\mathrm{ZnO}$ nanosheet network/anionic nanoclay layer heterojunction grown on the $\mathrm{Al}$ electrode. A schematic image of the nanogenerator fabricated with the 2D nanosheet network/anionic nanoclay layer heterojunction is illustrated in Fig. 2a. A nonlinear Schottky-like current-voltage curve is obtained from the nanosheet nanogenerator (see Supplementary Fig. S3). To investigate the performance of the nanosheet nanogenerator, a mechanical force stimulator was used to cyclically apply pushing forces to nanosheet-based nanogenerator. At an applied pushing force of $1.0 \mathrm{kgf}$, the output voltage and output current density reach up to about $0.44 \mathrm{~V}$ and $6.5 \mu \mathrm{A} / \mathrm{cm}^{2}$, respectively (Fig. 2b), which are much larger than those of the nanogenerators with DC output in the previous reports ${ }^{17,18,31,32}$. An increase in applied pushing force leads to larger power output. The obtained DC power density was about $2.86 \mu \mathrm{W} / \mathrm{cm}^{2}$ under the $1.0 \mathrm{kgf}$. When the applied pushing force was increased gradually up to $3.0 \mathrm{kgf}$, the power density is increased to $\sim 8.1 \mu \mathrm{W} / \mathrm{cm}^{2}$. A DC power density of $\sim 11.8 \mu \mathrm{W} / \mathrm{cm}^{2}$ was achieved under the force of $4.0 \mathrm{kgf}$.

Previous nanogenerators based on $1 \mathrm{D} \mathrm{ZnO}$ nanostructures standing on various substrates could not sustain above a critical force (that

a
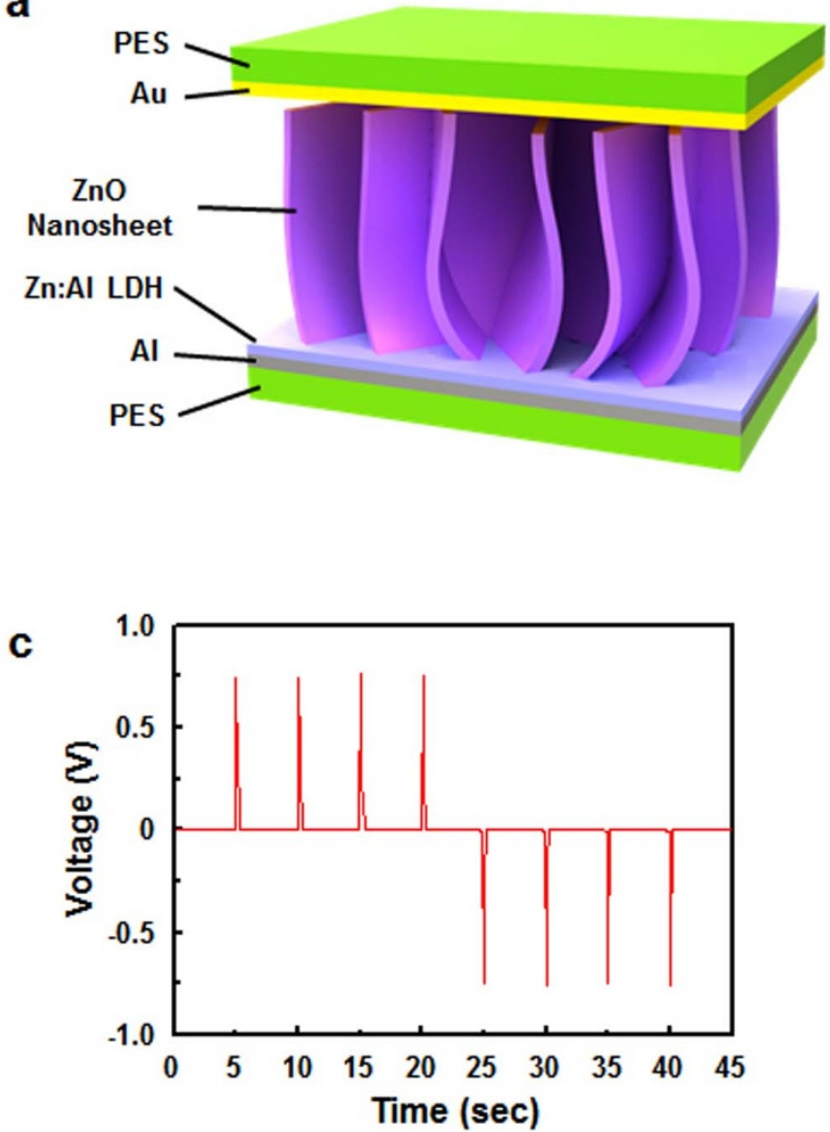

is certainly below this huge force of $4.0 \mathrm{kgf}$ ) due to the fracture of the nanostructures. However, this 2D nanosheet-based nanogenerator produces large output without any degradation in the device performance with such a huge force, indicating excellent mechanical durability of the device. In order to confirm that the measured output voltage and output current signals originate from the piezoelectric nanogenerator rather than detection noise, switching-polarity tests are performed to measure the voltage and current (Fig. $2 \mathrm{c}$ and $2 \mathrm{~d}$ ) by reversing the connection. The output voltage and output current density for both connecting conditions were about the same. A schematic illustration of the DC-type charge driving mechanism in the nanosheet-based nanogenerator is shown in Fig. 3. A large number of anions $\left(\mathrm{NO}_{3}{ }^{-}\right)$present in the $\mathrm{ZnAl}: \mathrm{LDH}$ layer at the interface of the $\mathrm{ZnO}$ nanosheets and the $\mathrm{Al}$ electrode play a major role in achieving DC output.

Proposed DC power generation mechanism of a piezoelectric nanogenerator fabricated with $2 \mathrm{D} \mathrm{ZnO}$ nanosheets/anionic layer heterojunction. The combined effect of buckling behaviour of the $\mathrm{ZnO}$ nanosheet, self-formed anionic nanoclay layer, and coupled semiconducting and piezoelectric properties of $\mathrm{ZnO}$ nanosheets contribute to efficient DC power generation. The initial stage illustration of the $2 \mathrm{D} \mathrm{ZnO}$ nanosheet-based nanogenerator with the Au top electrode is given in Fig. 3a. When $\mathrm{ZnO}$ nanosheet networks are subjected to an external force, the nanosheets are buckled and generate piezoelectric potential in the $\mathrm{ZnO}$ nanosheets. A positive potential is produced at the stretched side of the nanosheet, while a negative potential is induced at the compressed side of the nanosheet (Fig. 3b).

b
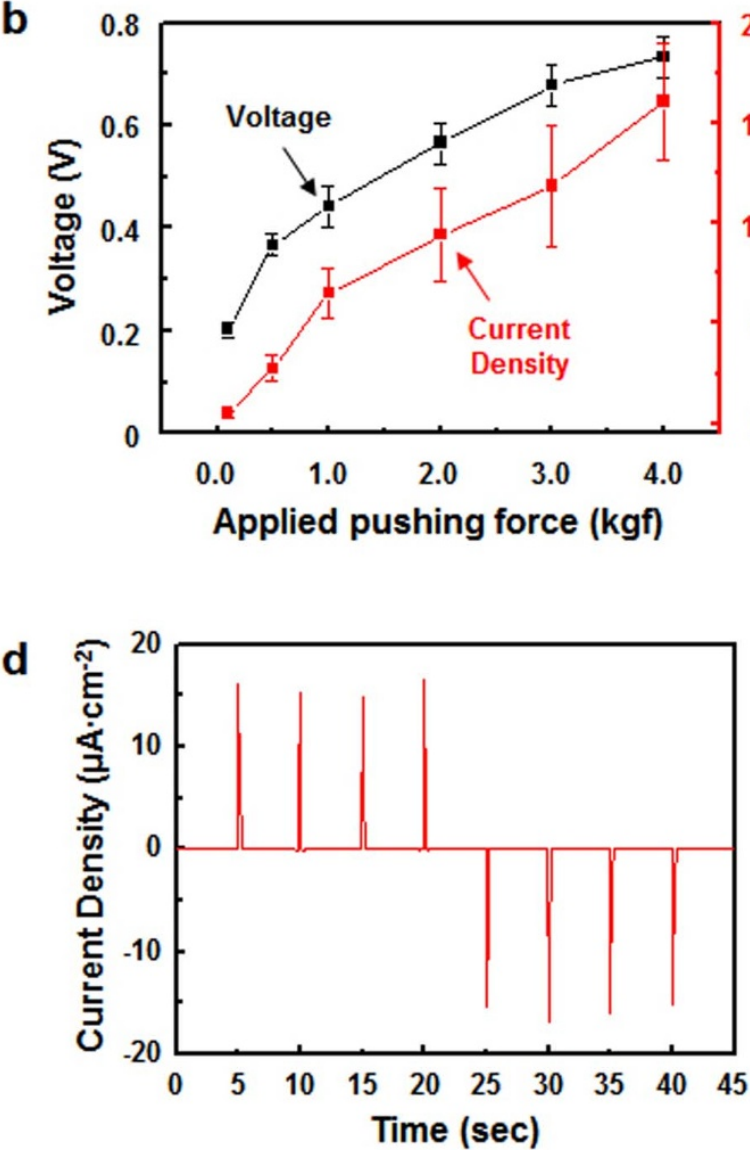

Figure $2 \mid$ Design and electrical output of a $\mathrm{ZnO}$ nanosheet-based nanogenerator. a, A schematic image of a $2 \mathrm{D} \mathrm{ZnO}$ nanosheet-based nanogenerator fabricated with the 2D nanosheet network/anionic nanoclay layer heterojunction. b, Output voltage and current density of $\mathrm{ZnO}$ nanosheet-based nanogenerator by varying the applied pushing force. An increase in applied pushing force leads to larger power output. A DC power density of $\sim 11.8 \mu \mathrm{W} /$ $\mathrm{cm}^{2}$ was achieved under the force of $4.0 \mathrm{kgf}$. c-d, are output voltage and output current density in switching-polarity tests at a load of $4.0 \mathrm{kgf}$, respectively. 
A Schottky contact forms at the interface of $\mathrm{Au}$ and $\mathrm{ZnO}$ nanosheets due to the larger work function of $\mathrm{Au}(5.1 \mathrm{eV})$ than the electron affinity of the $\mathrm{ZnO}(4.5 \mathrm{eV})$, which helps in preserving the piezoelectric potential. A piezoelectric potential generated in the $\mathrm{ZnO}$ nanosheets acts as a voltage applied for storing the charges from the $\mathrm{ZnO}$ nanosheets in the $\mathrm{LDH}$ layer, which act as a capacitor. Hence charges transported from the nanosheets are stored into the LDH layer under an applied external force as shown in Fig. 3b. The LDH layer containing positively charged layers as well as charge balancing anions $\left(\mathrm{NO}_{3}{ }^{-}\right)$located at the interlayer region is an anion exchange and weak conducting layer. The positive charges in the area of the $\mathrm{LDH}$ facing the nanosheets are compensated with free electrons from the $\mathrm{ZnO}$ nanosheets, as a result overall large negative

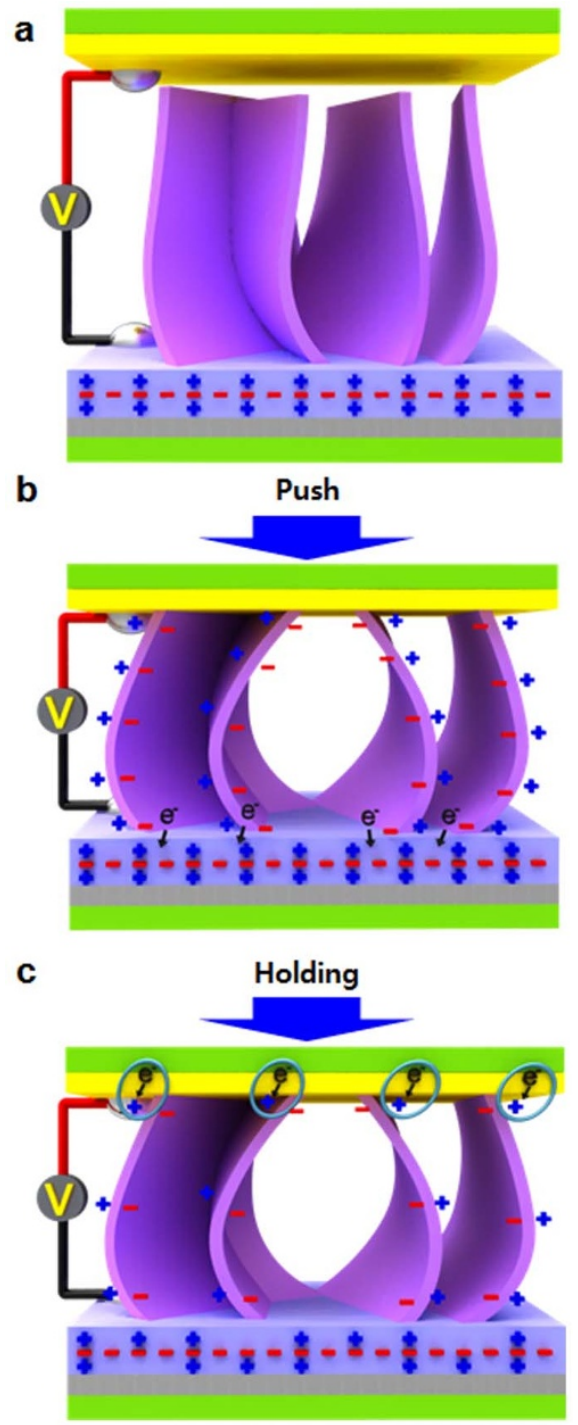

Figure $3 \mid$ Proposed mechanism for $\mathrm{DC}$ power generation from the $\mathrm{ZnO}$ nanosheet-based nanogenerator. a, A nanogenerator is electrically neutral under no external force. $\mathrm{b}$, When $\mathrm{ZnO}$ nanosheets are subjected to an external force, the nanosheets are buckled and generate piezoelectric potential. A positive potential is produced at the stretched side of the nanosheets and a negative potential is induced at the compressed side. Also, a positive charge layer of the $\mathrm{LDH}$ that is interfaced with nanosheets is compensated with the free electrons transported from the $\mathrm{ZnO}$ nanosheets. Consequently, the LDH layer is of a dominated negative potential. c, Electrons injected from the top electrode by overcoming a Schottky barrier between the top electrode and the $\mathrm{ZnO}$ nanosheets reduce the piezoelectric potential as time goes on at holding time. charges at the nanosheet/LDH interface build up. Subsequently, a strong potential difference is developed with the domination of negative charges in $\mathrm{LDH}$, which corresponds to the observed voltage pulses (Fig. 2) in the pushing force. The observed voltage pulse possesses the following behaviour (see Supplementary Fig. S4): The rising part of the pulse is ascribed to the pushing force, intermediate decaying part to holding time, and the decaying part of the ending pulse to releasing the force.

When the nanosheet-based nanogenerator starts experiencing an external pushing force (Fig. 3b), the nanosheets start buckling and generate piezoelectric potential corresponding to the measured rising part of the pulse. Once the force is held constant for a particular time (Fig. 3c) there is no further rise in piezoelectric potential, and then a positive potential in the $\mathrm{ZnO}$ nanosheets at the interface with the Au top electrode attracts electrons from the Au electrode. As a result, an existed piezoelectric potential starts to decrease, subsequently, and the pulse starts to decay with time. This process continues until the electrons screen the piezoelectric potential in the $\mathrm{ZnO}$ nanosheets. Consequently, although the device starts returning to the initial stage with holding time, but it could not return to initial state perfectly as it was before application of the external force. It is due to some small amount of piezoelectric potential still preserved in the holding time and could not be screened completely. As the external force is released, the device returns perfectly to the initial condition owing to the absence of the piezoelectric potential and full recovery of the $\mathrm{LDH}$ as a charge neutral layer. During this time, the decaying part of the ending pulse is measured. Only DC pulses of such behaviour are observed in each of the pushing and releasing forces. The generated current pulses from the device follow similar behaviour. A strong potential difference developed with the domination of negative charges at the $\mathrm{LDH} / \mathrm{Al}$ electrode; drive electrons from the bottom side of the $\mathrm{Al}$ electrode to the top side of the $\mathrm{Au}$ electrode. This is absolutely an observed reverse phenomenon of electrons transport start from the bottom electrode in comparison of observed in nanogenerators without $\mathrm{LDH}$, where electron transport process starts from the top electrode.

\section{Discussion}

We also investigated the performance of the $\mathrm{ZnO}$ nanosheet-based nanogenerator using several top electrodes of various work functions such as $\mathrm{Au}$ (Schottky contact with $\mathrm{ZnO}$ nanosheets), graphene (weak Schottky contact with $\mathrm{ZnO}$ nanosheets), indium tin oxide (ITO) (very weak Schottky contact with $\mathrm{ZnO}$ nanosheets), and $\mathrm{Al}$ (ohmic contact with $\mathrm{ZnO}$ nanosheets) (see Supplementary Figs. S5 and S6). The output performances of voltage and current pulses from the graphene, ITO, and Al top electrode-based nanogenerators are poor compared to that of the Au top electrode-based nanogenerator. It is due to lack and less preservation of piezoelectric potential in case of weak Schottky contact, very weak Schottky contact, and ohmic contact compared to preservation of piezoelectric potential in the case of good Schottky contact with the Au electrode which helps to store larger charges in the $\mathrm{LDH}$, resulting in stronger voltage and current pulses in response to applied force.

To clearly understand the mechanical stability of the $2 \mathrm{D} \mathrm{ZnO}$ nanosheet-based nanogenerator under an applied force, compression testing of $\mathrm{ZnO}$ nanosheets and $\mathrm{ZnO}$ nanorods grown on copper foil was performed with a nanoindentation system equipped with a $2 \mu \mathrm{m}$ diameter flat-punch type diamond tip. Compression testing up to $1 \mu \mathrm{m}$ from the top-end of the nanorods and nanosheets by nanoindentation gives insight into the $\mathrm{ZnO}$ nanorods and nanosheets which respond differently under uniaxial compression (Fig. 4a). Such response of nanorods and nanosheets under compression provides an intricate means of comparing mechanical properties of these two distinctively different types of nanostructures. Simple comparison of mechanical properties between two nanostructures by buckling forces or elastic modulus is not a proper method because 
force distribution on the two distinctive nanostructures is completely different. Furthermore, nanorods and nanosheets do not have completely normal to the surface as well as indentation direction, so compression force could not be uniformly distributed over all the structures under the flat-punch indenter tip. Hence, we adopted strain-energy density concept to understand the elastic stability of the two nanostructures. Since total energy introduced to the structures by compression will be absorbed by the structures in unit area, strain-energy density, which represents energy absorption ability until the structure is permanently deformed (in this case, abruptly changes in normal load) could be a representative property to explain how the materials respond to the external forces.

As indicated in Fig. 4a, abrupt changes in load-displacement curves of nanorods and nanosheets are presented by arrows, and named critical load $\left(\mathrm{P}_{\text {crt }}\right)$. We selected $\mathrm{P}_{\text {crt }}$ of nanorods at the largest increase in the displacement. Several 'pop-in' events (abrupt increases in the displacement) are observed before the critical load, $\mathrm{P}_{\text {crt }}$, which could be due to the buckling of the nanorods. It could not be the representative compressed force on nanorods by the indentation tip. We calculated the area under the curve before $\mathrm{P}_{\mathrm{crt}}$ to determine the strain-energy density of the nanorods. In the case of nanosheets, special care to select $\mathrm{P}_{\mathrm{crt}}$ is needed because it does not show a distinct change of load or displacement as shown in Fig. 4a. We differentiated the load-displacement curve, and we then selected an inflection point at the constriction of the structure. This force before constriction of the network structure of nanosheets could be used as the representative force for nanosheets under the indentation

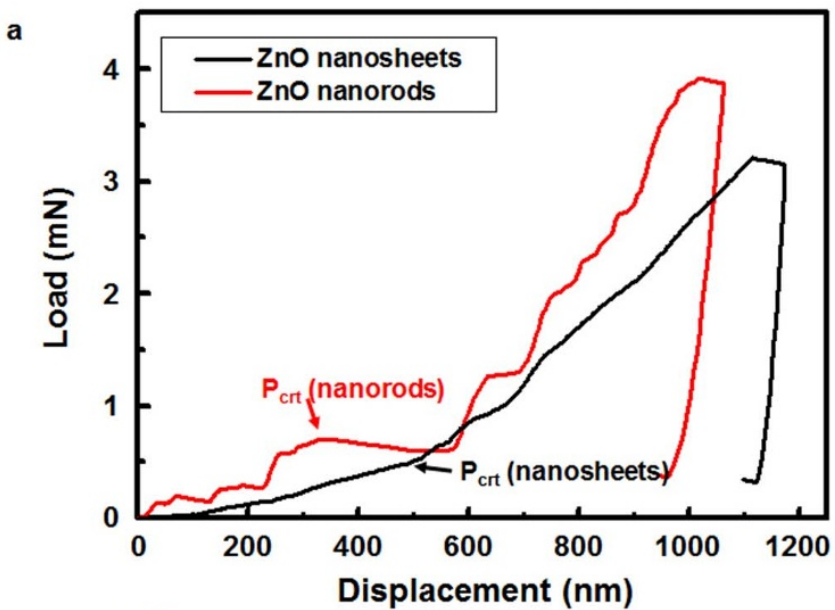

b

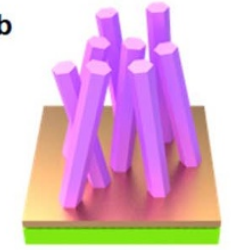

$$
\boldsymbol{P}<\boldsymbol{P}_{\text {crt }}
$$

e

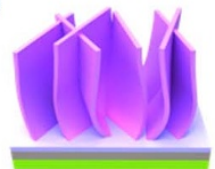

c

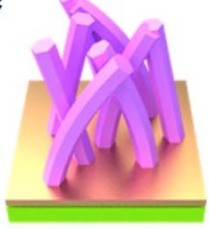

$\boldsymbol{P}=\boldsymbol{P}_{c r t}$

f

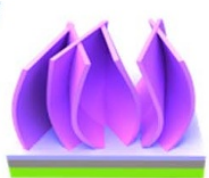

d

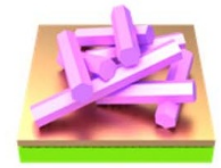

$\boldsymbol{P}>\boldsymbol{P}_{\text {crt }}$

g

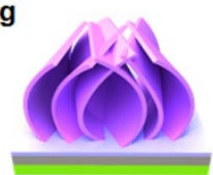

Figure $4 \mid$ Mechanical stability of $\mathrm{ZnO}$ nanorods and nanosheets. a, Load-displacement curve of $\mathrm{ZnO}$ nanosheets and nanorods using nanoindentor. Nanoindentation testing gives insight into the $\mathrm{ZnO}$ nanorods and nanosheets which respond differently under uniaxial compression. b-d and e-g, Schematic images describing the morphological development of $\mathrm{ZnO}$ nanorods and nanosheets, respectively, according to the applying load of below and above $\mathrm{P}_{\text {crt }}$.
Table 1 | The estimated strain-energy density of nanorods and nanosheets

\begin{tabular}{lcc} 
& ZnO Nanorods & ZnO Nanosheets \\
\hline Total Area $\left(\mathrm{m}^{2}\right)$ & $5.02 \times 10^{-13}$ & $3.83 \times 10^{-14}$ \\
Length $(\mathrm{m})$ & $2.00 \times 10^{-6}$ & $3.00 \times 10^{-6}$ \\
Density $\left(\mathrm{m}^{3}\right)$ & $1.00 \times 10^{-18}$ & $1.15 \times 10^{-19}$ \\
Strain-Energy Density $\left(\mathrm{J} / \mathrm{m}^{3}\right)$ & $7.88 \pm 2.19 \times 10^{6}$ & $8.20 \pm 1.77 \times 10^{7}$
\end{tabular}

tip. Since the strain energy $U$ of the nanostructures is distributed uniformly throughout its volume (under the flat-punch), it can be expressed as,

$$
U=\int_{0}^{\delta} P_{c r t} d \delta
$$

where $\delta$ is displacement. We can determine the strain-energy density by dividing the total strain energy with the volume of the structures. The estimated strain-energy density of the nanorods is about $7.88 \times$ $10^{6} \mathrm{~J} / \mathrm{m}^{3}$ whereas that of the nanosheets is $8.20 \times 10^{7} \mathrm{~J} / \mathrm{m}^{3}$ (Table 1 ). The strain-energy density of the $\mathrm{ZnO}$ nanosheets is about ten times larger than that of the the $\mathrm{ZnO}$ nanorods. The absorption of higher strain-energy density in the nanosheets is due to their networked structure.

In summary, we have demonstrated the first use of $2 \mathrm{D} \mathrm{ZnO}$ nanostructure and an anionic layer to generate large DC electrical output. The device made from such 2D piezoelectric nanosheets/anionic layer heterojunction has potential for use as a mechanically durable and smallest size power package, which can be used to charge wireless nano/micro-scale systems with no need for a rectifier circuit. The combined effect of buckling the $\mathrm{ZnO}$ nanosheets, anionic layer, and coupled semiconducting and piezoelectric properties of $\mathrm{ZnO}$ nanosheets are the main causes of the efficient DC power generation. Due to morphologically networked $\mathrm{ZnO}$ nanosheets, the $\mathrm{ZnO}$ nanosheets proved to be more structurally stable under huge external mechanical loads compared to $\mathrm{ZnO}$ nanorods. Furthermore, it was found that the $\mathrm{ZnO}$ nanosheet-based piezoelectric nanogenerators reveal much higher and more stable power-generating performance compared to previous piezoelectric DC output nanogenerators.

\section{Methods}

Growth of $\mathrm{ZnO}$ nanosheets/anionic layer heterojunction and integration of a nanogenerator. In order to grow $2 \mathrm{D} \mathrm{ZnO}$ nanosheets/anionic layer network, $\mathrm{Al}$ electrode was first cleaned using a buffered-oxide etch solution in order to remove native oxide on the $\mathrm{Al}$ surface. Then, $\mathrm{ZnO}$ nanosheets were grown via an aqueous solution route with a mixture solution consisting of zinc nitrate hexahydrate $\left[\mathrm{Zn}\left(\mathrm{NO}_{3}\right)_{2} \cdot 6 \mathrm{H}_{2} \mathrm{O}, 0.025 \mathrm{M}\right]$, hexamethylenetetramine $(0.025 \mathrm{M})$, and de-ionized water $(250 \mathrm{ml})$. The main growth of the $\mathrm{ZnO}$ nanosheets was carried out at $95^{\circ} \mathrm{C}$ for $3 \mathrm{hr}$. To fabricate the nanogenerators, a 100-nm-thick Au layer was deposited on PES substrates using a thermal evaporator as a top electrode which was integrated with $2 \mathrm{D}$ $\mathrm{ZnO}$ nanosheets.

Characterization and measurements. Morphological properties of the $\mathrm{ZnO}$ nanosheets were examined using FE-SEM (JOEL, JSM-7401F). The structural properties of the samples were determined by TEM (JOEL, JEM-2100F) and synchrotron XRD (Pohang Light Source) measurements. A picoammeter (Keithley 6485) and an oscilloscope (Tektronix DPO 3052) were used for low-noise current and voltage measurements to detect currents and voltages generated. To investigate the mechanical properties of the nanosheets network, compression testing was performed with a nanoindentation system (Nanoindenter G200, Agilient, USA) equipped with a $2 \mu \mathrm{m}$ diameter flat-punch type diamond tip (Synton-MDP Ltd., Swiss). More than 20 indentation tests were performed for each specimen.

1. Xu, S. et al. Self-powered nanowire devices. Nat. Nanotech. 5, 366-373 (2010).

2. Yang, R., Qin, Y., Dai, L. \& Wang, Z. L. Power generation with laterally packaged piezoelectric fine wires. Nat. Nanotech. 4, 34-39 (2009).

3. Cha, S. N. et al. Sound-driven piezoelectric nanowire-based nanogenerators. $A d v$. Mater. 22, 4726-4730 (2010). 
4. Choi, M. et al. Mechanically powered transparent flexible charge-generating nanodevices with piezoelectric ZnO nanorods. Adv. Mater. 21, 2185-2189 (2009).

5. Choi, D. et al. Fully rollable transparent nanogenerators based on graphene electrodes. Adv. Mater. 22, 2187-2192 (2010).

6. Lee, M. et al. A hybrid piezoelectric structure for wearable nanogenerators. $A d v$. Mater. 24, 1759-1764 (2012).

7. Qi, Y. \& McApline, M. C. Nanotechnology-enabled flexible and biocompatible energy harvesting. Energy Environ. Sci. 3, 1275-1285 (2010).

8. Lee, K. Y. et al. P-Type polymer-hybridized high-performance piezoelectric nanogenerators. Nano Lett. 12, 1959-1964 (2012).

9. Kumar, B. \& Kim, S.-W. Energy harvesting based on semiconducting piezoelectric ZnO nanostructures. Nano Energy 1, 342-355 (2012).

10. Chang, C., Tran, V. H., Wang, J., Fuh, Y.-K. \& Lin, L. Direct-write piezoelectric polymeric nanogenerator with high energy conversion efficiency. Nano Lett. 10, 726-731 (2010).

11. Qi, Y. et al. Enhanced piezoelectricity and stretchability in energy harvesting devices fabricated from buckled PZT ribbons. Nano Lett. 11, 1331-1336 (2011).

12. Xu, S., Hansen, B. J. \& Wang, Z. L. Piezoelectric-nanowire-enabled power source for driving wireless microelectronics. Nat. Commun. 1, 1-5 (2010).

13. Chen, X., Xu, S., Yao, N. \& Shi, Y. 1.6 V Nanogenerator for mechanical energy harvesting using PZT nanofibers. Nano Lett. 10, 2133-2137 (2010).

14. Jung, J. H. et al. Lead-free $\mathrm{NaNbO}_{3}$ nanowires for a high output piezoelectric nanogenerator. ACS Nano 5, 10041-10046 (2011).

15. Qi, Y. et al. Piezoelectric ribbons printed onto rubber for flexible energy conversion. Nano Lett. 10, 524-528 (2010).

16. Park, K.-I. et al. Piezoelectric $\mathrm{BaTiO}_{3}$ thin film nanogenerator on plastic substrates. Nano Lett. 10, 4939-4943 (2010).

17. Wang, Z. L. \& Song, J. H. Piezoelectric nanogenerators based on zinc oxide nanowire array. Science 312, 242-246 (2006).

18. Wang, X., Song, J., Liu, J. \& Wang, Z. L. Direct-current nanogenerator driven by ultrasonic waves. Science 316, 102-105 (2007).

19. Kumar, B. \& Kim, S.-W. Recent Advances in Power Generation through Piezoelectric Nanogenerators. J. Mater. Chem. 21, 18946-18958 (2011).

20. Novoselov, K. S. et al. Electric field effect in atomically thin carbon films. Science 306, 666-669 (2004)

21. Lee, K. H. et al. Large-scale synthesis of high-quality hexagonal boron nitride nanosheets for large-area graphene electronics. Nano Lett. 12, 714-718 (2012).

22. Radisavljevic, B., Radenovic, A., Brivio, J., Giacometti, V. \& Kis, A. Single-layer $\mathrm{MoS}_{2}$ transistors. Nat. Nanotechnol. 6, 147-150 (2011).

23. Coleman, J. N. et al. Two-dimensional nanosheets produced by liquid exfoliation of layered materials. Science 331, 586-571 (2011).

24. Kumar, B. et al. General route to single-crystalline $\mathrm{SnO}$ nanosheets on arbitrary substrates. J. Phys. Chem. C. 114, 11050-11055 (2010).

25. Guo, X., Zhang, F., Evans, D. G. \& Duan, X. Layered double hydroxide films: synthesis, properties and applications. Chem. Commun. 46, 5197-5210 (2010).

26. Lee, J. H., Rhee, S. W., Nam, H. J. \& Jung, D.-Y. Surface selective deposition of PMMA on layered double hydroxide nanocrystals immobilized on solid substrates. Adv. Mater. 21, 546-549 (2009).
27. Zhang, F. et al. Corrosion resistance of superhydrophobic layered double hydroxide films on aluminum. Angew. Chem. Int. Ed. 47, 2466-2469 (2008).

28. Lee, J. H., Rhee, S. W. \& Jung, D.-Y. Selective layer reaction of layer-by-layer assembled layered double-hydroxide nanocrystals. J. Am. Chem. Soc. 129, 3522 3523 (2007).

29. Yarger, M. S., Steinmiller, E. M. P. \& Choi, K.-S. Electrochemical synthesis of ZnAl layered double hydroxide (LDH) films. Inorg. Chem. 47, 5859-5865 (2008).

30. Shi, W., He, S., Wei, M., Evans, D. G. \& Duan, X. Optical pH sensor with rapid response based on a fluorescein-intercalated layered double hydroxide. $A d v$. Funct. Mater. 20, 3856-3863 (2010).

31. Park, H.-K. et al. Charge-generating mode control in high-performance transparent flexible piezoelectric nanogenerators. Adv. Funct. Mater. 21, 1187-1193 (2011).

32. Kumar, B. et al. Controlled growth of semiconducting nanowire, nanowall, and hybrid nanostructures on graphene for piezoelectric nanogenerators. ACS Nano 5, 4197-4204 (2011).

\section{Acknowledgments}

This work was financially supported by the National Research Foundation of Korea (NRF) grant funded by the Ministry of Education, Science and Technology (MEST) (2012R1A2A1A01002787, 2010-0019086, 2010-0015035, and 2011-0027090) and the Energy International Collaboration Research \& Development Program of the Korea Institute of Energy Technology Evaluation and Planning (KETEP) funded by the Ministry of Knowledge Economy (MKE) (2011-8520010050).

\section{Author contributions}

K.-H.K. and B.K. contributed equally to this work. Sample synthesis and electrical characterization were done by K.-H.K., B.K. and K.Y.L. FE-SEM, TEM and Raman measurements and analyses were performed by H.-K.P. and J.-H.L. The synchrotron XRD work was conducted by H.H.L. The nanoindentation tests of the samples and analysis were performed by H.J. and D.L. The project was planned by S.-W.K. All authors contributed to the manuscript writing.

\section{Additional information}

Supplementary information accompanies this paper at http://www.nature.com/ scientificreports

Competing financial interests: The authors declare no competing financial interests. How to cite this article: Kim, K.-H. et al. Piezoelectric two-dimensional nanosheets/anionic layer heterojunction for efficient direct current power generation. Sci. Rep. 3, 2017; DOI:10.1038/srep02017 (2013).

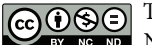

This work is licensed under a Creative Commons AttributionNonCommercial-NoDerivs Works 3.0 Unported license. To view a copy of this license, visit http://creativecommons.org/licenses/by-nc-nd/3.0 